\title{
EXPERIMENTAL INVESTIGATION ON MONOTONIC BEHAVIOR OF CIRCULAR STEEL STIFFENED COMPOSITE COLUMN UNDER COMPRESSION
}

\author{
Athar Nihal ${ }^{1}$, N.S Kumar ${ }^{2}$ \\ ${ }^{\text {I}}$ Master of Technology (Structural Engineering) Student, Civil Department, Ghousia College of Engineering, \\ Ramanagaram, Karnataka, India \\ ${ }^{2}$ Professor \& Director $(R \& D)$ Civil Department, Ghousia College of Engineering, Ramanagaram, Karnataka, India
}

\begin{abstract}
Concrete-filled square or rectangular thin-walled steel tubular columns are becoming increasingly attractive to be used in engineering practice. However, local buckling is more likely to occur for thin-walled tubes, and thus has adverse effects on strength and ductility for the composite columns. In order to improve their overall performance, one of the most effective stiffening measures is to provide longitudinal stiffeners for the steel tubes. In this experiment, Monotonic Behavior of Circular Steel Stiffened Composite Column under Compression is studied. In this study typical Fifty Four specimens of variable thickness $t_{1} \& t_{2}$ and variable dia $d_{1} \& d_{2}$ with piercing and also 54 specimens of thickness $t_{1} \& t_{2}$, and variable dia $d_{1} \& d_{2}$ without piercing is considered. For each Thickness combination of stiffeners at different position are selected that is for single stiffeners at mid span, from top $h / 3$, from bottom $h / 3$ and also for double stiffeners equispaced, from top $h / 2 \&$ from bottom $h / 3$, from top $h / 3 \&$ from bottom h/2. Experiments were carried out for four different cases : Hollow, Uncompacted Sand, Compacted Sand \& Dry Cement \& Dry Sand in order to know the behavior and buckling load of above said columns and tests were conducted under Monotonic loading.

Major conclusion from this study are: Load carrying capacity of tubes with single stiffener with piercing at mid height found to decrease by $8-10 \%$ than hollow tubes of same diameter and thickness, but for with single stiffener without piercing at mid height found to increase by $2 \%$ than hollow tubes of same diameter and thickness. Also, Load carrying capacity of tubes with single stiffener without piercing at mid height was found to increase by 10-15\% than pierced stiffener at mid height of same diameter and thickness.
\end{abstract}

Keywords: Composite, Monotonic,stiffner, buckling etc., ****.

\section{INTRODUCTION}

Concrete-filled steel tubular (CFST) columns have been increasingly used in many modern structures, such as dwelling houses, tall buildings, and arch bridges (Tao and $\mathrm{Yu}, 2006$; Uy and Patil, 2006; Han, 2007). This is due to the fact that CFST columns have high strength, high stiffness, and high ductility for optimal usage of construction materials, that is, the steel tube can confine the in filled concrete, and the concrete core can also restrain local buckling of the steel tube.

This research evaluates the seismic performance of circular concrete-filled tube (CCFT) columns in accelerated bridge construction (ABC) projects. Current $\mathrm{ABC}$ usually uses precast concrete columns grouted to rebar connections at base and top, if intermediate columns are required. The bridge can be assembled in a few days, but the seismic performance objectives cannot be reached until the columns' top and base connection grout reaches design strength. The advantage of CCFT columns is the use of standard bolted connection at the top and bottom of the column - capable of resisting design loads upon being bolted without the need to wait for curing design strength to be reached. The bolted connection also eliminates rebar congestion at the connection. Also, the materials needed to construct CCFT columns are readily available. The time the CCFT concrete filling takes to cure, and the column's reduced capacity for that duration, poses a primary challenge when CCFT columns are considered for ABC. This study investigates whether a designation of temporary condition can be used to reduce the Design Basis Earthquake (DBE).

Steel-concrete composite columns were used for over a century. At the beginning it was used to provide fire protection to steel structures. Afterwards, the concrete encased columns strength properties were also considered in the design. However, the researches into concrete filled steel tubes (CFST) did not begin until the 1960[1]. Nowadays, the composite structural elements are increasingly used in tall buildings, bridges and other types of structures. The steel concrete composites are considered as an advantageous system for carrying large axial load benefitting from the interaction between the concrete and the steel section.

The steel section reinforces the concrete to resist any bending moments, tensile and shear forces. The concrete in a composite column reduces the potential for buckling of the steel section in addition to resisting compressive loading. 
Concrete Filled Steel Tubes (CFSTs) are used in many structure application including column, supporting platform of offshore structures, roofs of storage tanks, bridge piers, and column in seismic zones. Concrete filled steel box column offer excellent structural performance, such as high strength, high ductility and large energy absorption capacity. Application of the CFST concept can lead to over all saving of steel in comparison with conventional structural steel systems. In CFST composite construction, steel tubes are also used as permanent formwork and to provide well distribution reinforcement.

Composite columns are structural members, which are subjected mainly to axial compressive forces and end moments. The general term 'composite column' refers to any compression member in which the steel element acts compositely with the concrete as shown in fig 1 . so that both elements contribute to the strength. These columns have been used widespread as they speed up construction by eliminating formwork and the need for tying of longitudinal reinforcement. Composite columns have recently undergone increased usage throughout the world, which has been influenced by the development of high strength concrete permitting these columns to be considerably economized. Columns designed to resist the majority of axial force by concrete alone can be further economized by the use of thinwalled steel columns. New developments, including the use of high strength concrete and the credit of the enhanced local buckling capacity of the steel has allowed much more economical designs to evolve. The main economy achieved by using high strength concrete in thin steel casings is that the structural steel cost is minimized and the majority of the load in compression is resisted by the high strength concrete. Furthermore, the concrete is enhanced in its performance as it suffers less creep and shrinkage and the quality improves, thus allowing a larger compressive stress to be resisted by the internal concrete. Conditions of the applied load are defined as axial, uniaxial and biaxial. This type of behaviour is possible in concrete filled steel columns in multi-storey building.

\subsection{Stiffened Steel Tubes}

Steel tubes of uniform length are stiffened at different position are selected that is for single stiffeners at mid span, from top $h / 3$, from bottom $h / 3$ and also for double stiffeners equispaced,from top $\mathrm{h} / 2 \&$ from bottom $\mathrm{h} / 3$, from top $\mathrm{h} / 3$ \&from bottom $\mathrm{h} / 2$.

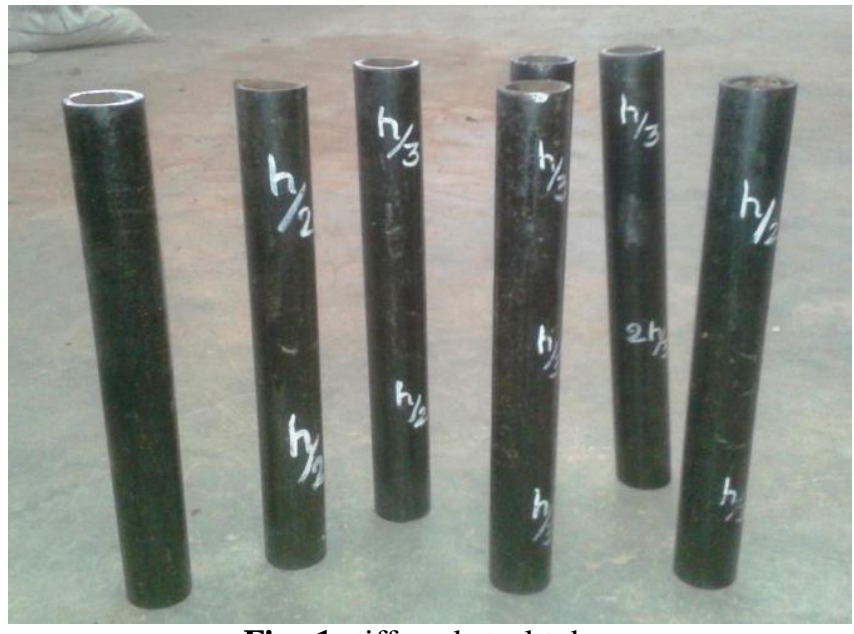

Fig:-1 stiffened steel tubes

\section{METHODOLOGY}

The following specimens are tested using in UTM by studying the behavior of the column section to determine buckling load and deformation for various types of the section or specimens of two different thicknesses $t_{1} \& t_{2}$ given below.

\section{By Piercing}

1. Hollow steel tubes - (7specimens)

2. Uncompacted sand - (7specimens)

3. Compacted sand $-(7$ specimens $)$

4. Dry cement and sand - (6specimens)

\section{Without Piercing}

1. Hollow steel tubes $-(7$ specimens $)$

2. Uncompacted sand - (7specimens)

3. Compacted sand - (7specimens $)$

4. Dry cement and sand - (6specimens)

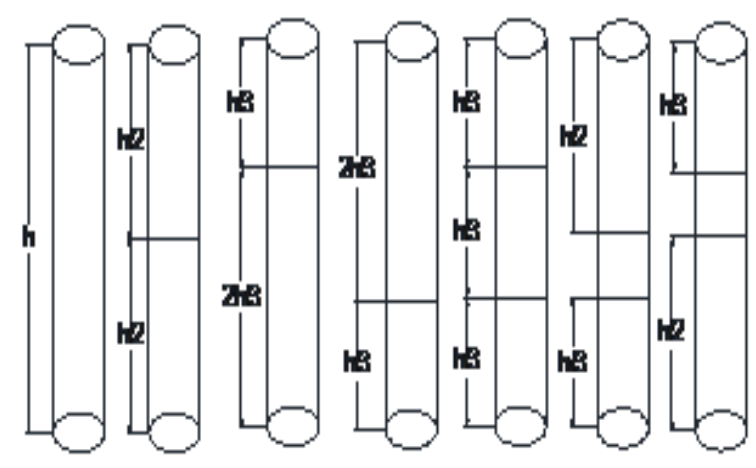

Fig-2 placement of stiffeners at different positions

Monotonic loading is carried out for all the specimen to determined strength \& deformation. 


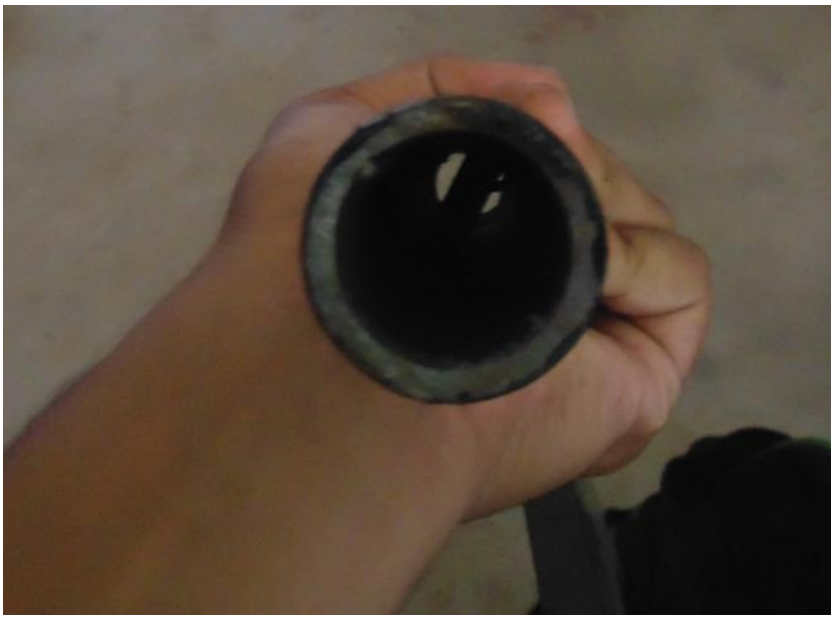

Fig2.4: steel tube with stiffner

\section{EXPERIMENTAL RESULTS AND} DISCUSION

Steel Specimens: In this study following different thickness and different diameter with same length specimens are selected as listed in table1, table2, table3

1. Hollow stiffened steel tubes - (table1)

2. Uncompacted sand filled stiffened steel tubes-(table2)

3. Compacted sand filled stiffened steel tubes-(table3)

4. Dry cement and dry sand filled steel tubes-(table4)

Table 1

\begin{tabular}{|c|c|c|c|c|c|}
\hline \multirow[t]{4}{*}{ SI no } & \multirow{4}{*}{$\begin{array}{l}\text { Hollow } \\
\text { tubes }\end{array}$} & \multicolumn{4}{|c|}{ ULTIMATE LOAD Pu } \\
\hline & & \multicolumn{2}{|c|}{ With piercing } & \multicolumn{2}{|c|}{$\begin{array}{l}\text { Without } \\
\text { piercing }\end{array}$} \\
\hline & & $\begin{array}{l}\mathrm{D}_{1}=27 \\
\mathrm{~mm}\end{array}$ & $\begin{array}{l}\mathrm{D}_{2}=28 \\
\mathrm{~mm}\end{array}$ & $\begin{array}{l}\mathrm{D}_{1}=27 \\
\mathrm{~mm}\end{array}$ & $\begin{array}{l}\mathrm{D}_{2}=28 \\
\mathrm{~mm}\end{array}$ \\
\hline & & $\begin{array}{l}\mathrm{T}_{1}=4 \\
\mathrm{~mm}\end{array}$ & $\begin{array}{l}\mathrm{T}_{2}=3 \mathrm{~m} \\
\mathrm{~m}\end{array}$ & $\begin{array}{l}\mathrm{T}_{1}=4 \mathrm{~m} \\
\mathrm{~m}\end{array}$ & $\begin{array}{l}\mathrm{T}_{2}=3 \mathrm{~m} \\
\mathrm{~m}\end{array}$ \\
\hline 1 & hallow & $\begin{array}{l}127.5 \\
\mathrm{KN}\end{array}$ & $82 \mathrm{KN}$ & $\begin{array}{l}127.5 \\
\mathrm{KN}\end{array}$ & $82 \mathrm{KN}$ \\
\hline 2 & $\begin{array}{l}\text { 1- } \\
\text { stiffner }\end{array}$ & $\begin{array}{l}115 \mathrm{~K} \\
\mathrm{~N}\end{array}$ & $65 \mathrm{KN}$ & $130 \mathrm{KN}$ & $65 \mathrm{KN}$ \\
\hline 3 & $\begin{array}{l}1- \\
\text { stiffner } \\
\text { From } \\
\text { top } \mathrm{h} / 3\end{array}$ & $\begin{array}{l}115 \mathrm{~K} \\
\mathrm{~N}\end{array}$ & $80 \mathrm{KN}$ & $\begin{array}{l}127.5 \\
\mathrm{KN}\end{array}$ & $80 \mathrm{KN}$ \\
\hline 4 & $\begin{array}{l}\text { 1- } \\
\text { stiffner } \\
\text { form } \\
\text { bottom } \\
\text { h/3 } \\
\end{array}$ & $\begin{array}{l}132.5 \\
\mathrm{KN}\end{array}$ & $\begin{array}{l}77.5 \mathrm{~K} \\
\mathrm{~N}\end{array}$ & $\begin{array}{l}142.5 \\
\mathrm{KN}\end{array}$ & $\begin{array}{l}77.5 \mathrm{~K} \\
\mathrm{~N}\end{array}$ \\
\hline 5 & $\begin{array}{l}\text {-- } \\
\text { stiffner } \\
\text { equispac } \\
\text { ed }\end{array}$ & $\begin{array}{l}115.5 \\
\mathrm{KN}\end{array}$ & $\begin{array}{l}62.5 \mathrm{~K} \\
\mathrm{~N}\end{array}$ & $\begin{array}{l}132.5 \\
\mathrm{KN}\end{array}$ & $\begin{array}{l}62.5 \mathrm{~K} \\
\mathrm{~N}\end{array}$ \\
\hline 6 & $\begin{array}{l}\text { 2- } \\
\text { stiffners } \\
\text { from }\end{array}$ & $\begin{array}{l}125 \mathrm{~K} \\
\mathrm{~N}\end{array}$ & $80 \mathrm{KN}$ & $140 \mathrm{KN}$ & $80 \mathrm{KN}$ \\
\hline
\end{tabular}

\begin{tabular}{|l|l|l|l|l|l|}
\hline & $\begin{array}{l}\text { bottom } \\
\text { h/3 }\end{array}$ & & & & \\
\hline 7 & $\begin{array}{l}\mathbf{2}- \\
\text { stiffners } \\
\text { from top } \\
\text { h/3 }\end{array}$ & 112.5 & $60 \mathrm{KN}$ & $\begin{array}{l}122.5 \\
\mathrm{KN}\end{array}$ & $60 \mathrm{KN}$ \\
\hline
\end{tabular}

Table 2

\begin{tabular}{|c|c|c|c|c|c|}
\hline \multirow{4}{*}{$\begin{array}{l}\text { S } \\
\text { l } \\
\text { n } \\
\text { o }\end{array}$} & \multirow{4}{*}{$\begin{array}{l}\text { Uncompact } \\
\text { ed Sand }\end{array}$} & \multicolumn{4}{|c|}{ ULTIMATE LOAD $P_{u}$} \\
\hline & & \multicolumn{2}{|c|}{ With piercing } & \multicolumn{2}{|c|}{$\begin{array}{l}\text { Without } \\
\text { piercing }\end{array}$} \\
\hline & & $\begin{array}{l}\mathrm{D}_{1}=27 \\
\mathrm{~mm}\end{array}$ & $\begin{array}{l}\mathrm{D}_{2}=28 \\
\mathrm{~mm}\end{array}$ & $\begin{array}{l}\mathrm{D}_{1}=27 \\
\mathrm{~mm}\end{array}$ & $\begin{array}{l}D_{2}=2 \\
8 \mathrm{~mm}\end{array}$ \\
\hline & & $\begin{array}{l}\mathrm{T}_{1}=4 \mathrm{~m} \\
\mathrm{~m}\end{array}$ & $\begin{array}{l}\mathrm{T}_{2}=3 \mathrm{~m} \\
\mathrm{~m}\end{array}$ & $\begin{array}{l}\mathrm{T}_{1}=4 \mathrm{~m} \\
\mathrm{~m}\end{array}$ & $\begin{array}{l}\mathrm{T}_{2}=3 \\
\mathrm{~mm}\end{array}$ \\
\hline 1 & hallow & $\begin{array}{l}107.5 \mathrm{~K} \\
\mathrm{~N}\end{array}$ & $65 \mathrm{KN}$ & $\begin{array}{l}107.5 \mathrm{~K} \\
\mathrm{~N}\end{array}$ & $\begin{array}{l}65 \mathrm{~K} \\
\mathrm{~N}\end{array}$ \\
\hline 2 & 1-stiffner & $115 \mathrm{KN}$ & $66 \mathrm{KN}$ & $131 \mathrm{KN}$ & $\begin{array}{l}99 \mathrm{~K} \\
\mathrm{~N}\end{array}$ \\
\hline 3 & $\begin{array}{l}\text { 1-stiffner } \\
\text { From top } \\
\text { h/3 }\end{array}$ & $\begin{array}{l}127.5 \mathrm{~K} \\
\mathrm{~N}\end{array}$ & $80 \mathrm{KN}$ & $142 \mathrm{KN}$ & $\begin{array}{l}95 \mathrm{~K} \\
\mathrm{~N}\end{array}$ \\
\hline 4 & $\begin{array}{l}\text { 1-stiffner } \\
\text { form } \\
\text { bottom h/3 }\end{array}$ & $130 \mathrm{KN}$ & $100 \mathrm{KN}$ & $144 \mathrm{KN}$ & $\begin{array}{l}98 \mathrm{~K} \\
\mathrm{~N}\end{array}$ \\
\hline 5 & $\begin{array}{l}\text { 2-stiffner } \\
\text { equispaced }\end{array}$ & $112 \mathrm{KN}$ & $83 \mathrm{KN}$ & $\begin{array}{l}127.5 \mathrm{~K} \\
\mathrm{~N}\end{array}$ & $\begin{array}{l}95 \mathrm{~K} \\
\mathrm{~N} \\
\end{array}$ \\
\hline 6 & $\begin{array}{l}\text { 2-stiffners } \\
\text { from } \\
\text { bottom } \mathbf{h} / 3\end{array}$ & $125 \mathrm{KN}$ & $80 \mathrm{KN}$ & $140 \mathrm{KN}$ & $\begin{array}{l}99 \mathrm{~K} \\
\mathrm{~N} \\
\end{array}$ \\
\hline 7 & $\begin{array}{l}\text { 2-stiffners } \\
\text { from top } \\
\text { h/3 }\end{array}$ & $110 \mathrm{KN}$ & $66 \mathrm{KN}$ & $120 \mathrm{KN}$ & $\begin{array}{l}101 \mathrm{~K} \\
\mathrm{~N}\end{array}$ \\
\hline
\end{tabular}

Table 3

\begin{tabular}{|c|c|c|c|c|c|}
\hline \multirow{4}{*}{$\begin{array}{l}\text { Sl } \\
\text { n } \\
\text { o }\end{array}$} & \multirow{4}{*}{$\begin{array}{l}\text { Compact } \\
\text { ed Sand }\end{array}$} & \multicolumn{4}{|c|}{ ULTIMATE LOAD $P_{u}$} \\
\hline & & \multicolumn{2}{|c|}{ With piercing } & \multicolumn{2}{|c|}{ Without piercing } \\
\hline & & $\begin{array}{l}\mathrm{D}_{1}=27 \mathrm{~m} \\
\mathrm{~m}\end{array}$ & $\begin{array}{l}\mathrm{D}_{2}=28 \mathrm{~m} \\
\mathrm{~m}\end{array}$ & $\begin{array}{l}\mathrm{D}_{1}=27 \mathrm{~m} \\
\mathrm{~m}\end{array}$ & $\begin{array}{l}\mathrm{D}_{2}=28 \mathrm{~m} \\
\mathrm{~m}\end{array}$ \\
\hline & & $\begin{array}{l}\mathrm{T}_{1}=4 \mathrm{~m} \\
\mathrm{~m}\end{array}$ & $\begin{array}{l}\mathrm{T}_{2}=3 \mathrm{~m} \\
\mathrm{~m}\end{array}$ & $\begin{array}{l}\mathrm{T}_{1}=4 \mathrm{~m} \\
\mathrm{~m}\end{array}$ & $\begin{array}{l}\mathrm{T}_{2}=3 \mathrm{~m} \\
\mathrm{~m}\end{array}$ \\
\hline 1 & hallow & $130 \mathrm{KN}$ & $65 \mathrm{KN}$ & $130 \mathrm{KN}$ & $65 \mathrm{KN}$ \\
\hline 2 & 1-stiffner & $110 \mathrm{KN}$ & $67.5 \mathrm{KN}$ & $135 \mathrm{KN}$ & $98 \mathrm{KN}$ \\
\hline 3 & $\begin{array}{l}\text { 1-stiffner } \\
\text { From } \\
\text { top h/3 }\end{array}$ & $\begin{array}{l}127.5 \mathrm{~K} \\
\mathrm{~N}\end{array}$ & $77.5 \mathrm{KN}$ & $142 \mathrm{KN}$ & $92.5 \mathrm{KN}$ \\
\hline 4 & $\begin{array}{l}\text { 1-stiffner } \\
\text { form } \\
\text { bottom } \\
\text { h/3 }\end{array}$ & $\begin{array}{l}127.5 \mathrm{~K} \\
\mathrm{~N}\end{array}$ & $77.5 \mathrm{KN}$ & $142 \mathrm{KN}$ & $102 \mathrm{KN}$ \\
\hline 5 & $\begin{array}{l}\text { 2-stiffner } \\
\text { equispac } \\
\text { ed }\end{array}$ & $110 \mathrm{KN}$ & $67.5 \mathrm{KN}$ & $137 \mathrm{KN}$ & $100 \mathrm{KN}$ \\
\hline
\end{tabular}




\begin{tabular}{|l|l|l|l|l|l|}
\hline 6 & $\begin{array}{l}\mathbf{2 -} \\
\text { stiffners } \\
\text { from } \\
\text { bottom } \\
\text { h/3 }\end{array}$ & $127.5 \mathrm{~K}$ & $100 \mathrm{KN}$ & $144 \mathrm{KN}$ & $115 \mathrm{KN}$ \\
\hline 7 & $\begin{array}{l}\mathbf{2 -} \\
\text { stiffners } \\
\text { from top } \\
\text { h/3 }\end{array}$ & $110 \mathrm{KN}$ & $65 \mathrm{KN}$ & $142 \mathrm{KN}$ & $97 \mathrm{KN}$ \\
\hline
\end{tabular}

Table 4

\begin{tabular}{|c|c|c|c|c|c|}
\hline \multirow{4}{*}{$\begin{array}{l}\text { S } \\
\text { l } \\
\text { n } \\
\text { o }\end{array}$} & \multirow{4}{*}{$\begin{array}{l}\text { Mortar } \\
\text { filled } \\
\text { Hollow } \\
\text { tubes }\end{array}$} & \multicolumn{4}{|c|}{ ULTIMATE LOAD $P_{u}$} \\
\hline & & \multicolumn{2}{|c|}{ With piercing } & \multicolumn{2}{|l|}{$\begin{array}{l}\text { Without } \\
\text { piercing }\end{array}$} \\
\hline & & $\begin{array}{l}\mathrm{D}_{1}=27 \\
\mathrm{~mm}\end{array}$ & $\begin{array}{l}\mathrm{D}_{2}=28 \\
\mathrm{~mm}\end{array}$ & $\begin{array}{l}\mathrm{D}_{1}=27 \\
\mathrm{~mm}\end{array}$ & $\begin{array}{l}\mathrm{D}_{2}=28 \\
\mathrm{~mm}\end{array}$ \\
\hline & & $\begin{array}{l}\mathrm{T}_{1}=4 \mathrm{~m} \\
\mathrm{~m}\end{array}$ & $\begin{array}{l}\mathrm{T}_{2}=3 \mathrm{~m} \\
\mathrm{~m}\end{array}$ & $\begin{array}{l}\mathrm{T}_{1}=4 \mathrm{~m} \\
\mathrm{~m}\end{array}$ & $\begin{array}{l}\mathrm{T}_{2}=3 \mathrm{~m} \\
\mathrm{~m}\end{array}$ \\
\hline 1 & $\begin{array}{l}\text { Cement= } \\
50 \% \\
\text { Sand=50 } \\
\%\end{array}$ & $\begin{array}{l}137.5 \mathrm{~K} \\
\mathrm{~N}\end{array}$ & $\begin{array}{l}77.5 \mathrm{~K} \\
\mathrm{~N}\end{array}$ & $\begin{array}{l}137.5 \mathrm{~K} \\
\mathrm{~N}\end{array}$ & $\begin{array}{l}77.5 \mathrm{~K} \\
\mathrm{~N}\end{array}$ \\
\hline 2 & $\begin{array}{l}\text { Cement= } \\
25 \% \\
\text { Sand }=75 \\
\%\end{array}$ & $\begin{array}{l}142.5 \mathrm{~K} \\
\mathrm{~N}\end{array}$ & $\begin{array}{l}77.5 \mathrm{~K} \\
\mathrm{~N}\end{array}$ & $\begin{array}{l}142.5 \mathrm{~K} \\
\mathrm{~N}\end{array}$ & $\begin{array}{l}77.5 \mathrm{~K} \\
\mathrm{~N}\end{array}$ \\
\hline 3 & $\begin{array}{l}\text { Cement }= \\
75 \% \\
\text { Sand }=25 \\
\%\end{array}$ & $\begin{array}{l}127.5 \mathrm{~K} \\
\mathrm{~N}\end{array}$ & $80 \mathrm{KN}$ & $\begin{array}{l}127.5 \mathrm{~K} \\
\mathrm{~N}\end{array}$ & $80 \mathrm{KN}$ \\
\hline
\end{tabular}

Table 5

\begin{tabular}{|c|c|c|c|c|c|}
\hline \multirow{4}{*}{$\begin{array}{l}\text { S } \\
\text { l } \\
\text { n } \\
\text { o }\end{array}$} & \multirow{4}{*}{$\begin{array}{l}\text { Mortar } \\
\text { filled } \\
\text { 1-stiffner } \\
\text { tubes }\end{array}$} & \multicolumn{4}{|c|}{ ULTIMATE LOAD $P_{u}$} \\
\hline & & \multicolumn{2}{|c|}{ With piercing } & \multicolumn{2}{|c|}{ Without piercing } \\
\hline & & $\begin{array}{l}D_{1}=27 m \\
m\end{array}$ & $\begin{array}{l}\mathrm{D}_{2}=28 \\
\mathrm{~mm}\end{array}$ & $\begin{array}{l}\mathrm{D}_{1}=27 \\
\mathrm{~mm}\end{array}$ & $\begin{array}{l}\mathrm{D}_{2}=28 \\
\mathrm{~mm}\end{array}$ \\
\hline & & $\begin{array}{l}\mathrm{T}_{1}=4 \mathrm{~m} \\
\mathrm{~m}\end{array}$ & $\begin{array}{l}\mathrm{T}_{2}=3 \mathrm{~m} \\
\mathrm{~m}\end{array}$ & $\begin{array}{l}\mathrm{T}_{1}=4 \mathrm{~m} \\
\mathrm{~m}\end{array}$ & $\begin{array}{l}\mathrm{T}_{2}=3 \mathrm{~m} \\
\mathrm{~m}\end{array}$ \\
\hline 1 & $\begin{array}{l}\text { Cement=5 } \\
0 \% \\
\text { Sand=50 } \\
\%\end{array}$ & $\begin{array}{l}127.5 .5 \\
\mathrm{KN}\end{array}$ & $80 \mathrm{KN}$ & $140 \mathrm{KN}$ & $92 \mathrm{KN}$ \\
\hline 2 & $\begin{array}{l}\text { Cement=2 } \\
5 \% \\
\text { Sand=75 } \\
\%\end{array}$ & $\begin{array}{l}102.5 \mathrm{~K} \\
\mathrm{~N}\end{array}$ & $77.5 \mathrm{KN}$ & $\begin{array}{l}117.5 \mathrm{~K} \\
\mathrm{~N}\end{array}$ & $92.5 \mathrm{KN}$ \\
\hline 3 & $\begin{array}{l}\text { Cement=7 } \\
5 \% \\
\text { Sand=25 } \\
\%\end{array}$ & $125 \mathrm{KN}$ & $77.5 \mathrm{KN}$ & $137 \mathrm{KN}$ & $90 \mathrm{KN}$ \\
\hline
\end{tabular}

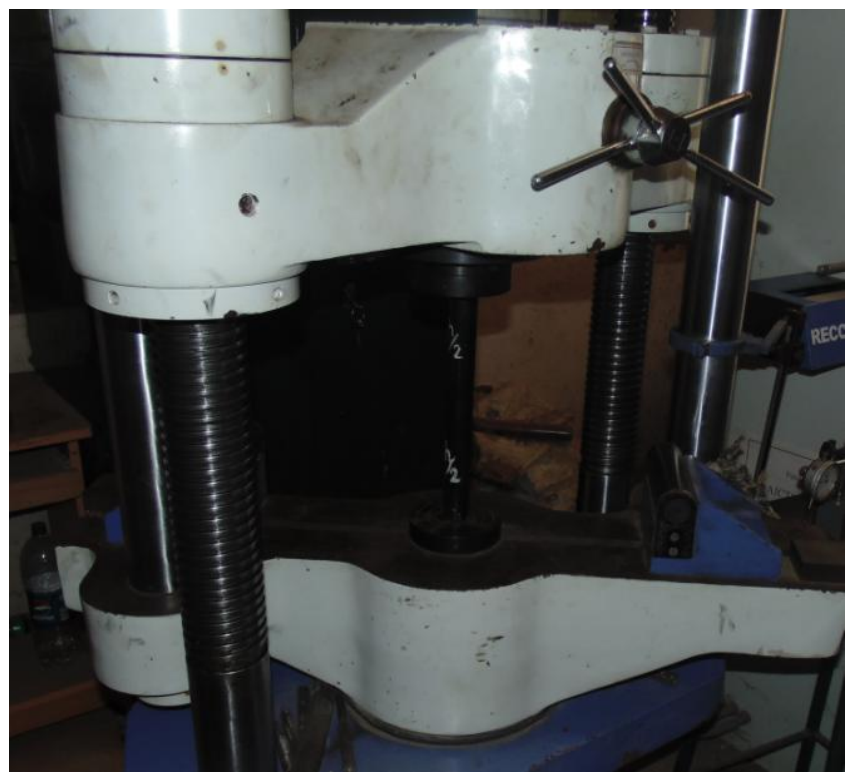

Fig 3.2: before testing of stiffened steel tube

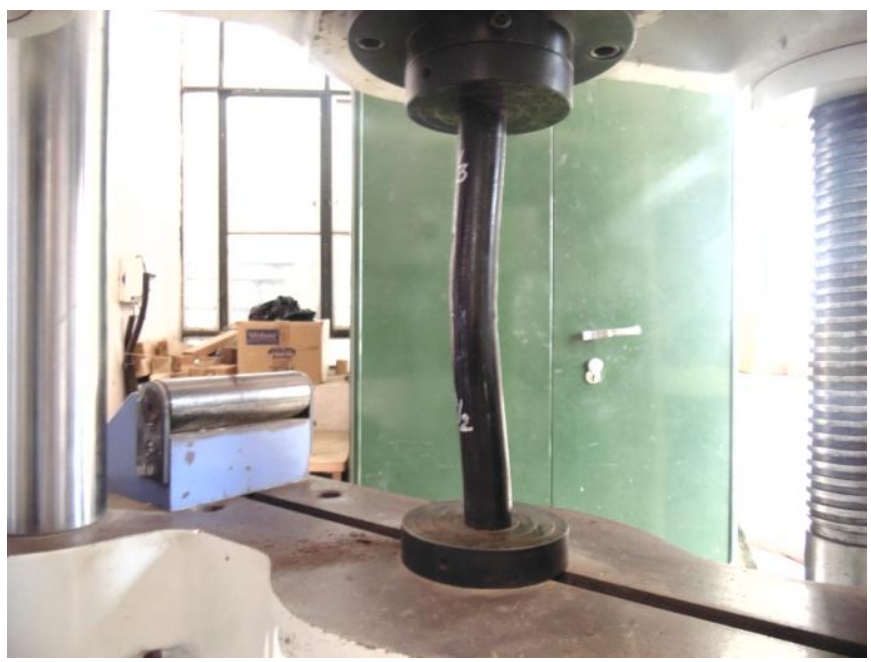

Fig 3.3: After testing Steel tube (Buckling observed at Middle of Steel tube)

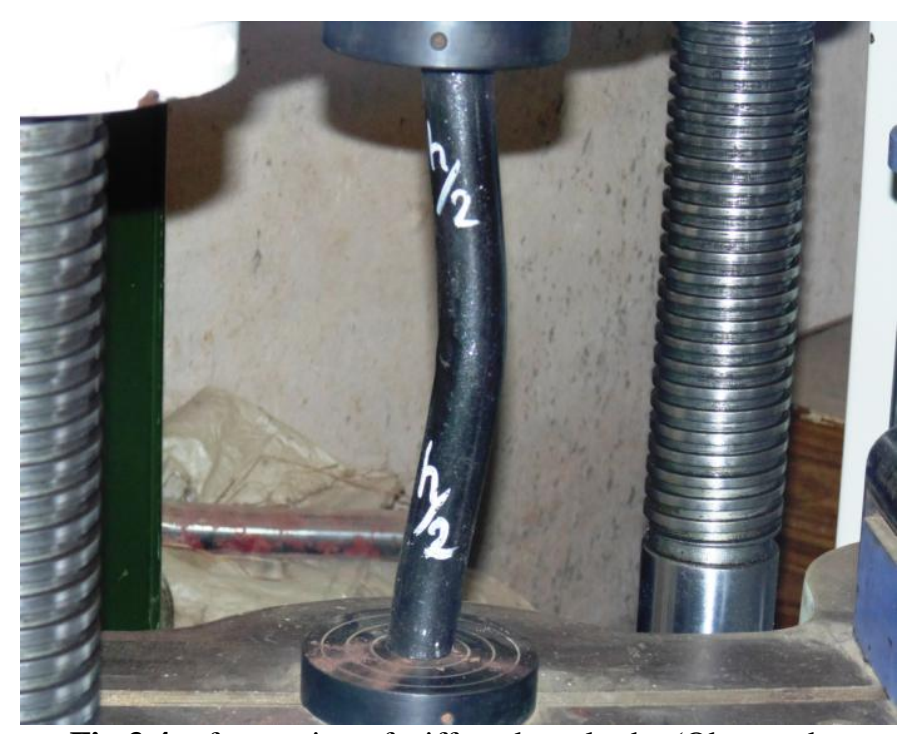

Fig 3.4: after testing of stiffened steel tube (Observed buckling at middle of steel tubes but stiffer is unaffected) 


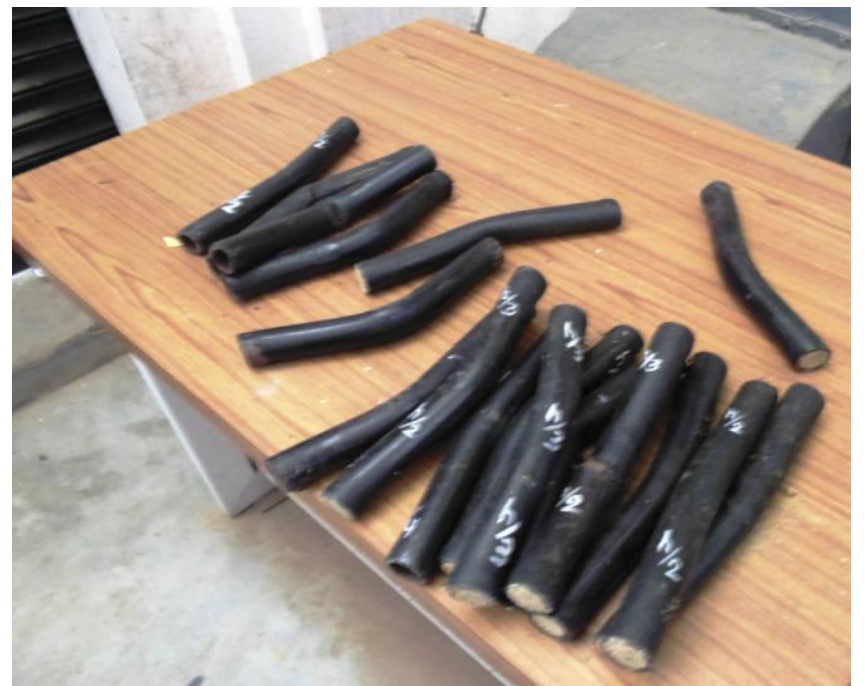

Fig 3.6: Steel column specimens after testing

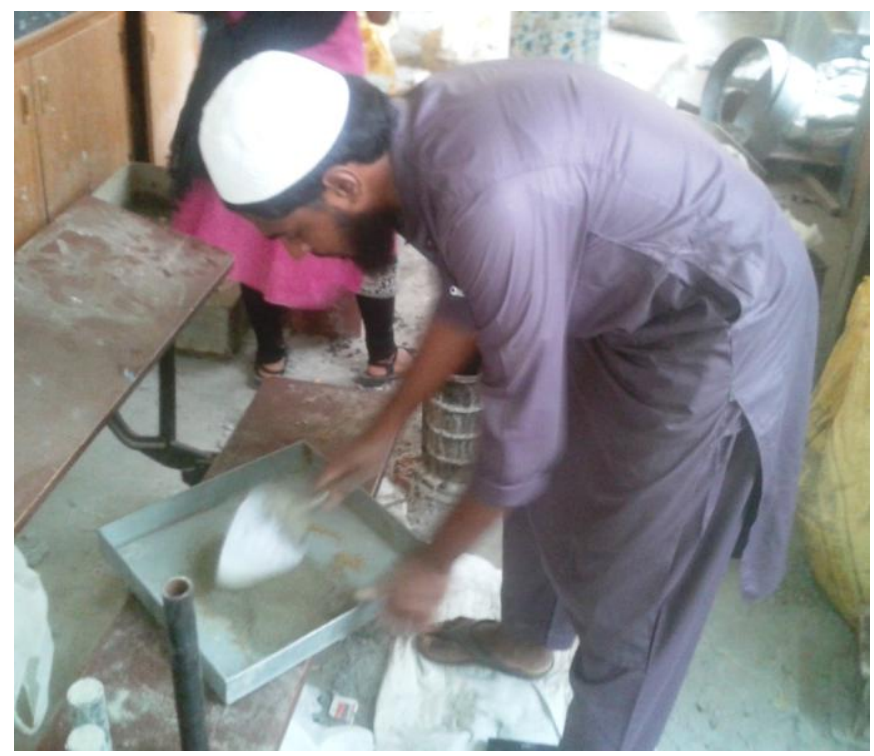

Fig 3.7: mixing of sand and cement with corresponding ratios

\subsection{Grapical Representation}

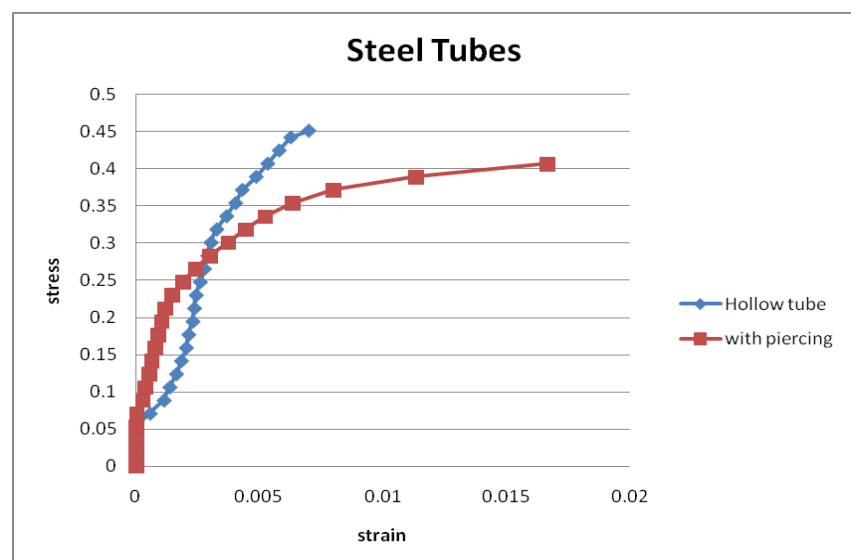

Fig 3.1: comparison $b / w$ hollow tube with 1-stiffner at mid height (with piercing)

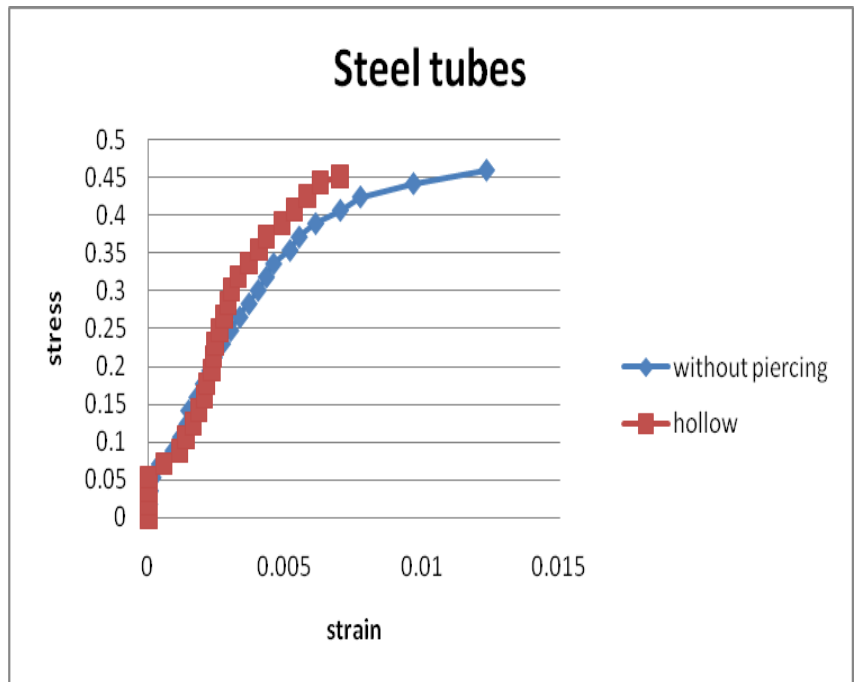

Fig 3.2: comparison $b / w$ hollow tube with 1-stiffner at mid height (without piercing)

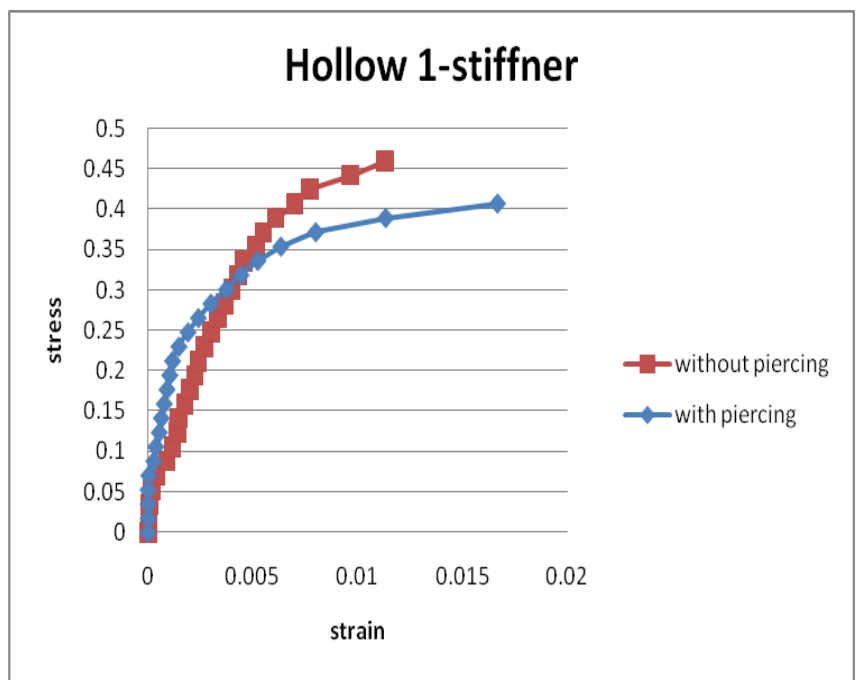

Fig 3.3: comparison $b / w$ hollow tube 1-stiffner at mid height (with piercing) with 1-stiffner at mid height (without piercing)

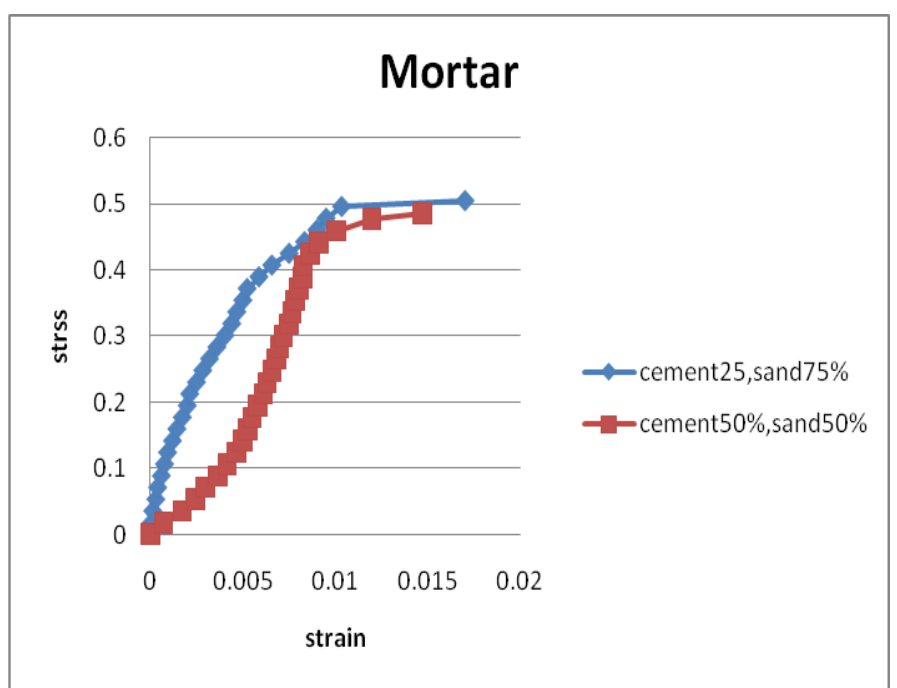

Fig 3.4: comparison of hollow tube filled with mortar having cement $50 \%$, sand $50 \%$ with cement $25 \%$,sand $75 \%$ 


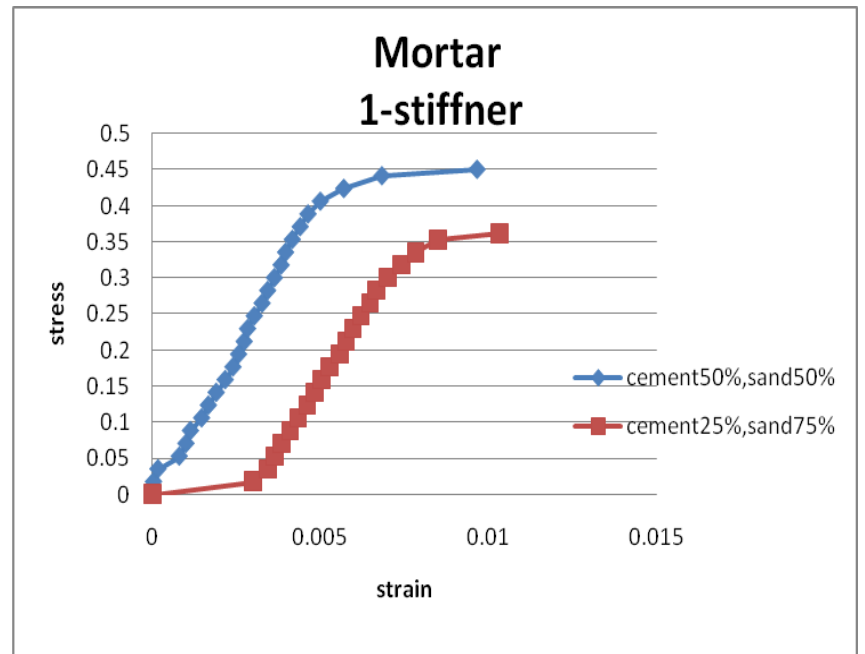

Fig 3.5: comparison of 1-stiffned hollow tube filled with mortar having cement $50 \%$, sand $50 \%$ with cement $25 \%$, sand $75 \%$

\section{CONCLUSION}

- Load carrying capacity of tubes with single stiffener with piercing at mid height found to decrease by 8 $10 \%$ than hollow tubes of same dia and thickness e.g.:(127.5kn,115kn)

- Load carrying capacity of tubes with single stiffener without piercing at mid height found to increase by $2 \%$ than hollow tubes of same dia and thickness e.g.:(127.5kn,130kn)

- Load carrying capacity of tubes with single stiffener without piercing at mid height was found to increase by $10-15 \%$ than pierced stiffener at mid height of same dia and thickness e.g.:(127.5kn, $115 \mathrm{kn}$

\section{REFERENCES}

[1]. Tao, Z., Han, L. H., and Wang, Z. B. (2005). "Experimental behaviors of stiffened concrete-filled thinwalled hollow steel structural (HSS) stub columns." Journal of Constructional Steel Research, 61, pp. 962-983.

[2]. Elremaily A, Azizinamini A. Behavior and strength of circular concrete-filled tube columns. Journal of Constructional Steel Research 2002;58(12):1567-91.

[3]. Hajjar J. Concrete-filled steel tube columns under earthquake loads. J. Progress Struct. Engng Mater. 2000;2(1):1-10.

[4]. Sakino K, Tomii M. Hysteretic behavior of concrete filled square steel tubular beam-columns failed in flexure. Trans. of the Japan Concrete Institute, 1981, vol.3: 439-46. [5]. Wang, W., L. Han and B. Uy, 2008. Experimental behaviour of steel reduced beam section to concrete-filled circular hollow section column connections. Journal of Constructional Steel Research, 64:493-504.

\section{BIOGRAPHIES}

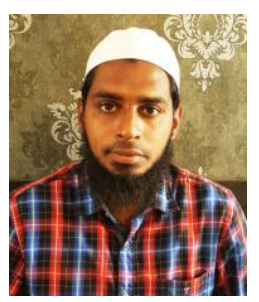

Athar Nihal, Obtained B.E degree in Civil Engineering (Second Class) during the year 2012 from Government Engineering College k.r.pet Affiliated to VTU Belgaum.. Presently perusing Master of Technology in Structural Engineering at Ghousia College of Engineering, Ramanagaram Also working on this topic for the dissertation under the guidance of Dr. N S Kumar.

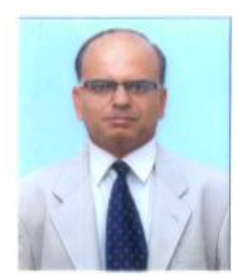

N.S Kumar, Involved in the Research field related to behavior of Composite Steel Column since a decade. \& He has guided more than 15 M.tech projects including one M.Sc Engineering (by Research under VTU, Belgaum). Presently guiding four Ph.D Scholars under VTU Belgaum. Has more than 26 years of teaching \& 6 years of Research experience at Ghousia College of Engineering, Ramanagaram 